\title{
High-level expression of Egr-1 and Egr-1-inducible genes in mouse and human atherosclerosis
}

\author{
Timothy A. McCaffrey, ${ }^{1}$ Chenzhong Fu, ${ }^{1}$ Baoheng Du, ${ }^{1}$ Sukru Eksinar, ${ }^{1} \mathrm{~K}$. Craig Kent, ${ }^{2}$ \\ Harry Bush, Jr., ${ }^{2}$ Karl Kreiger, ${ }^{3}$ Todd Rosengart, ${ }^{3}$ Myron I. Cybulsky, ${ }^{4}$ \\ Eric S. Silverman, ${ }^{5}$ and Tucker Collins ${ }^{5}$ \\ ${ }^{1}$ Department of Medicine, Division of Hematology/Oncology, \\ ${ }^{2}$ Department of Surgery, Division of Vascular Surgery, and \\ ${ }^{3}$ Department of Cardiothoracic Surgery, Weill Medical College of Cornell University, New York, New York 10021, USA \\ ${ }^{4}$ Department of Laboratory Medicine and Pathobiology, Toronto General Research Institute, University of Toronto, \\ Ontario M5G 2C4, Canada \\ ${ }^{5}$ Department of Pathology, Brigham and Women's Hospital, Harvard Medical School, Boston, Massachusetts 02115, USA \\ Address correspondence to: Timothy A. McCaffrey, Weill Medical College of Cornell University, 1300 York Avenue C-608, \\ New York, New York 10021, USA. Phone: (212) 746-2089; Fax: (212) 746-8866; E-mail: tamccaf@med.cornell.edu.
}

Received for publication October 1, 1999, and accepted in revised form January 11, 2000.

\begin{abstract}
To understand the mRNA transcript profile in the human atherosclerotic lesion, RNA was prepared from the fibrous cap versus adjacent media of 13 patients undergoing carotid endarterectomy. cDNA expression arrays bearing 588 known genes indicated that lesions express unexpectedly high levels of the early growth response gene, Egr-1 (NGFI-A), a zinc-finger transcription factor that modulates a cluster of stress-responsive genes including PDGF and TGF- $\beta$. Expression of Egr- 1 was an average of 5 -fold higher in the lesion than in the adjacent media, a result confirmed by RT-PCR, and many Egr1-inducible genes were also strongly elevated in the lesion. Time-course analyses revealed that Egr-1 was not induced ex vivo. Immunocytochemistry indicated that Egr-1 was expressed prominently in the smooth muscle-actin positive cells, particularly in areas of macrophage infiltration, and in other cell types, including endothelial cells. Induction of atherosclerosis in LDL receptor-null mice by feeding them a high-fat diet resulted in a progressive increase in Egr-1 expression in the aorta. Thus, induction of Egr-1 by atherogenic factors may be a key step in coordinating the cellular events that result in vascular lesions.
\end{abstract}

J. Clin. Invest. 105:653-662 (2000).

\section{Introduction}

The early growth response gene-1 (Egr-1) was identified as an important modulator in diverse cell types and thus has acquired several names, including: nerve growth factor-induced-A (NGFI-A), Krox-24, ZIF268, ETR103, and TIS8 (reviewed in refs. 1-3). Egr-1 is an $80-82-\mathrm{kD}$ inducible protein that is a prototype of the early growth response gene family, including Egr-2 and Egr-3. Members of the family have been implicated in commitments to proliferate, differentiate, or engage cell death pathways in vascular and other cell types. Egr-1 is rapidly and transiently induced by a variety of extracellular stimuli, including growth factors and cytokines, hypoxia, physical forces, and injurious stimuli. Egr-1 is a zinc-finger, DNA-binding protein that interacts with a consensus GC-rich region, GCG(T/G)GGGCG, to influence the transcription of a diverse set of genes. Egr-1 has been implicated in the induced expression of growth factors such as PDGF-A chain $(4,5)$, PDGF-B chain (6), basic fibroblast growth factor (bFGF; FGF-2) (7), and TGF- $\beta$ (8). Cytokines, such as TNF- $\alpha$ (9), and adhesion molecules, such as intercellular adhesion molecule 1 (ICAM-1) (10), and CD44 (11) can be affected. Cell-cycle modulators such as p53 can be induced (12). Regulatory components of the coagulation system, such as tissue factor (13) and urokinase-type plasminogen activator (u-PA) $(6,14)$, as well as metalloproteinases (15), are influenced by Egr1. Initial studies with mice having a targeted mutation in Egr-1 suggest that some of these genes are authentic targets for this transcription factor (16).

After acute arterial injury, a series of cellular changes occur in the vessel wall that often results in pathology. These changes result, at least in part, from the proteins that are inducibly expressed by injured endothelium and smooth muscle cells (SMC). Previous studies in animal models of arterial injury demonstrated that elevated Egr-1 levels can be found at sites of vascular injury, and it was suggested that inducible Egr- 1 expression in response to vessel injury may coordinate the expression of multiple genes involved in the pathogenesis of vascular disease (6). In more recent studies, it was demonstrated that Egr-1 induction in endothelial cells after injury was triggered by release and paracrine activation by FGF-2 (17). Synthetic DNA enzymes that degrade the Egr-1 mRNA reduce SMC migration and proliferation and reduce intimal hyperplasia after balloon-catheter injury in the rat (18). These findings suggest that Egr- 
1-mediated gene transcription plays a key role in orchestrating the functional characteristics of the vessel wall after arterial injury.

Atherosclerosis is believed currently to be an excessive fibroproliferative response to chronic vascular injury by diverse agents. The pioneering work of Dr. Russell Ross, and many others, described the involvement of peptide growth factors and cytokines, such as PDGF and FGF, in orchestrating migration, proliferation, and matrix production by SMC-like cells, macrophages, lymphocytes, and endothelial cells (19). Other platelet-derived factors, such as TGF- $\beta$, act as autocrine/paracrine cellular products with multiple effects on proliferation, migration, and matrix synthesis. The coordinate regulation of these growth factor and cytokine signals is probably a key determinant of the cellular events that occur during atherogenesis. This regulation may be mediated by alterations in the amount or localization of various transcription factors within the lesion cells. In the course of screening the mRNA expression pattern of human atherosclerotic lesions using cDNA arrays, it was observed that Egr-1 levels often exceeded the levels of constitutive genes, such as actin, and that Egr-1 transcript levels were much higher in the lesion than the adjacent media. The present studies examined the expression of Egr-1- and Egr-1-responsive genes in these lesions. Elevated levels of Egr-1 were associated with increases in Egr-1 target genes. Immunocytochemistry was used to determine the expression pattern in the human carotid atherosclerotic lesion. Atherosclerosis in the LDL receptor-null mouse was also associated with increased Egr-1 expression. Collectively, these results implicate Egr-1 induction, and subsequent target gene expression, in human and mouse atherogenesis.

\section{Methods}

Tissue specimens. Vascular specimens were obtained during surgical revascularization procedures at The New York Presbyterian/Cornell Medical Center (New York, New York, USA) as waste surgical specimens under institutional review board-approved protocols. Surgical endarterectomy of carotid artery disease produces full diameter lesions of $2-5 \mathrm{~cm}$ in length that commonly contain tunica media, without adventitia. The medial tissue can be cleanly dissected from the overlying lesion. Internal mammary arteries were obtained as excess waste from coronary artery bypass grafting (CABG) operations.

Timeline of postsurgical sample processing. Under normal conditions, the specimens could be obtained in saline, returned to the laboratory, dissected under sterile conditions, and placed into RNAzol within 40 to $60 \mathrm{~min}$ utes of surgical excision. To investigate the possibility that early response genes were induced ex vivo within this time frame, dissection of the lesion was conducted within the surgical suite, and the specimens could be placed into RNAzol within 5 minutes. Parallel portions of tissue were then held in saline for 1 hour before being placed in RNAzol.
Immunocytochemistry. Portions of endarterectomy specimens were equilibrated with $30 \%$ sucrose and then embedded and sectioned in OCT. Frozen sections were immunostained with an Egr-1-specific antibody (C-19; Santa Cruz Biotechnology, Inc., Santa Cruz, California, USA) at a 1:50 dilution by standard procedures for the avidin-biotin-enhanced immunoperoxidase detection (Vector Laboratories, Burlingame, California, USA). Positive immunostaining was detected with diaminobenzidine tetrahydrochloride (DAB) and counterstained with hematoxylin.

In some patients $(n=4)$, sections were double-stained with rabbit polyclonal antibodies to Egr-1, detected by avidin-biotin peroxidase and DAB substrate (brown), and counterstained with mAbs to marker antigens detected with alkaline phosphatase-labeled secondary antibodies and NBT/BCIP substrate, producing a blue product. Cross-reactivity of the secondary antibodies (anti-rabbit and anti-mouse) was excluded with controls that employed only 1 antibody and a nonimmune antibody of the other species. Cell type-specific antibodies included SMC-specific $\alpha$-actin (Clone 1A4; Sigma Chemical Company, St. Louis, Missouri, USA) and macrophage-specific (HAM56; DAKO Corp. Carpinteria, California, USA) and lymphocyte-specific leukocyte common antigen (LCA) (CD45; DAKO).

$R N A$ purification. Tissues were transiently immersed (3 minutes) in RNAlater (Ambion Inc., Austin, Texas, USA) to minimize degradation and stabilize the RNA. Total RNA was purified from vascular specimens or LDC using RNAzol with minor modifications of the manufacturer's method. In particular, early precipitation reactions could not be performed on ice, as specified, because of excessive precipitation of polysaccharides from the lesions. RNA quantity and quality was assessed by agarose gel electrophoresis and ethidium bromide staining.

$R T-P C R$. RNA $(1-5 \mu \mathrm{g})$ was reversed transcribed with Moloney murine leukemia virus (MMLV) reverse transcriptase (Perkin-Elmer, Foster City, USA) using random hexamer primers. A portion of this cDNA (250-500 ng) was then used for PCR with Taq (GeneAmp; PerkinElmer) under the following conditions $\left(95^{\circ} \mathrm{C}\right.$ denaturing temperature for 1 minute, $60^{\circ} \mathrm{C}$ anneal/extend for $25-40$ cycles, with a final extension of $72^{\circ} \mathrm{C}$ for 10 minutes). Primers for Egr-1 were directed at 1847-1868 and 2371-2392 of the cDNA (GenBank M62829), producing a 545 -bp product. Human $\beta$-actin mRNA was detected with primers directed at 484-504 and 813-833 (GenBank AB004047), producing a 350-bp product. Human RhoA (GenBank L25080) was detected with primers annealing to $290-317$ and 545-572, producing a 283-bp product.

Genomic expression arrays. For genomic analysis, RNA was further purified by retention on glass fiber columns (HighPure; Boehringer-Mannheim, Indianapolis, Indiana, USA), DNAse digestion, ethanol precipitation, and a final gel filtration on a desalting column (Chroma-spin 200 CLONTECH Laboratories Inc., Palo Alto, California, USA) to remove small 
oligonucleotides or contaminants. The paired RNAs (i.e., media versus lesion) were reversed transcribed with MMLV, ${ }^{32} \mathrm{P}-\mathrm{ATP}$, and a mixture of 588 sequencespecific primers, as described by the manufacturer (CLONTECH). In some studies, the reverse transcription-labeling step was performed with Superscript (GIBCO BRL, Gaithersburg, Maryland, USA), an RNAse H-MMLV mutant that appeared to produce more efficient and consistent labeling. The labeled cDNAs were desalted to remove free isotope and then hybridized overnight to identical cDNA arrays under stringent conditions $\left(68^{\circ} \mathrm{C}\right)$. The array membranes were washed with $2 \times$ SSC with $1 \%$ SDS for $30 \mathrm{~min}$ utes, 4 times, and $0.1 \times$ SSC with $0.5 \%$ SDS for $30 \mathrm{~min}$ utes, 3 times, all at $68^{\circ} \mathrm{C}$. The paired membranes were exposed to storage phosphor plates (Eastman Kodak Co. Scientific Imaging Systems, New Haven, Connecticut, USA) for 1-5 days and quantified on a phosphoimager (Storm; Molecular Dynamics, Sunnyvale, California, USA).

Mice, diet, and immunohistochemical analysis. Low density lipoprotein (LDL) receptor-negative $\left(\mathrm{LDLR}^{-/}\right)$mice were created by homologous recombination as described (20). Mice were placed on a high-cholesterol diet consisting of $1.25 \%$ cholesterol (including $7.5 \%$ cocoa butter, $7.5 \%$ casein, and $0.5 \%$ cholic acid). Mice were sacrificed after $0,5,10$, and 20 weeks on the diet. These time points were selected based on preliminary studies that demonstrated lesions begin to develop in the aortic arch and near the ostia after 4 weeks on the diet. After sacrifice, the arterial tree was perfused with PBS $(40 \mathrm{~mL} / 10 \mathrm{~min})$ and then perfusion-fixed with buffered formaldehyde $(70 \mathrm{~mL} / 15-20 \mathrm{~min})$ at a pressure of $100 \mathrm{mmHg}$. Samples of the aorta were embedded in OCT and immunostained with the Egr-1 antibody as described above, using a fast red substrate for the peroxidase-labeled secondary antibody. Sections were lightly counterstained with methylene green.

\section{Results}

Expression of Egr-1 $m R N A$ and related genes in buman atherosclerotic carotid arteries: Analysis by genomic expression arrays. To understand the physiological and pathological state of the cells that compose the fibroproliferative component of the atherosclerotic lesion, tissues from the fibrous cap of lesions were compared with the adjacent, underlying tunica media of 13 patients with obstructive carotid atherosclerosis. RNA was simultaneously reverse transcribed, labeled radioactively, and then hybridized to spatially addressable cDNA arrays (Figure 1a). The results were quantified by Storm phosphoimager, the background subtracted, and the counts normalized by an average of 3 housekeeping genes (GAPDH, $\beta$-actin, and 23kDa highly basic protein; Figure $1 \mathrm{~b}$ ).

The overall results of the studies showed that the level of gene expression in lesion tissue was significantly correlated with expression in the tunica media $(r=0.87, P<0.05)$, an outcome consistent with both tissues possessing many similar basic functions. Quantitation of the arrays proved accurate and reliable: re-exposure of the same cDNA membrane for 1 day versus 4 days, followed by quantitation of each membrane, revealed a strong positive correlation $(r=$ $0.9991, P<0.001)$.

One surprising outcome from this genomic analysis was the relatively high-level expression of the Egr-1 mRNA transcript, a gene previously thought to be associated with the early growth response. Egr-1 mRNA levels were extremely high in the atherosclerotic lesion: on average they were 2 -fold higher (200\%) than the housekeeping genes ( $n=13$ patients, 3 experiments, range $=101-285 \%$ ). Levels were consistently lower in the adjacent media: an average of only $42 \%$ of housekeeping genes (range 9-71\%). Thus, there was an almost 5-fold induction of Egr-1 expression in the atherosclerotic lesion (Figure $1 \mathrm{~b}$ ).

\section{Figure 1}

Analysis of mRNA levels in human atherosclerotic lesions by cDNA arrays. The mRNA was purified from the fibrous cap (lesion) or the tunica media (media) of 13 patients and analyzed in pooled groups by hybridization to cDNA arrays. (a) Storage phosphor image of 1 array containing 588 unique cDNAs doublespotted onto nitrocellulose and hybridized with ${ }^{32} \mathrm{P}$-labeled, reverse-transcribed cDNA from human media acquired by endarterectomy of 6 pooled patients. (b) Quantitation from the storage phosphor screen was normalized to a mean housekeeping value (mean), which was the average of GAPDH, $\beta$-actin, and $23-\mathrm{kD}$ HBP for each array. Thirteen patients were analyzed in groups on 3 array pairs (media versus lesion). Error bars, SEM; $n=3$ experiments. $\mathrm{HBP}$, highly basic protein.
}

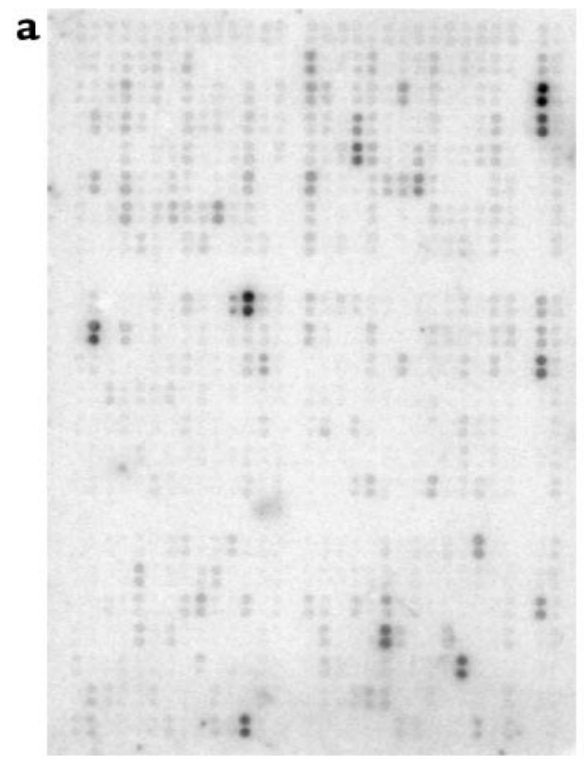




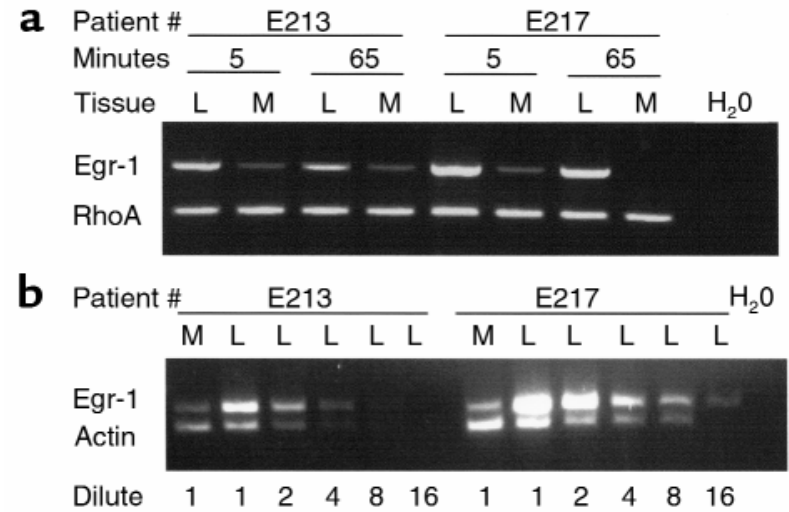

Figure 2

Analysis of mRNA levels in human lesion by RT-PCR. (a) The mRNA was prepared from the dissected fibrous cap of the lesion $(L)$ or the adjacent tunica media (M) within 5 minutes of surgical excision or after allowing an identical portion to incubate under transfer conditions for 65 minutes. The mRNAs were then analyzed by RT-PCR with primers directed at Egr-1 or RhoA. Two patients (E213, E217) are shown, demonstrating the difference noted in 3 of the 4 patients analyzed. (b) The difference in mRNA levels between media and lesion tissue was semiquantitated by serial dilution of the $L$ mRNA before RT-PCR for Egr-1 (upper band) or $\beta$-actin (lower band). Nonspecific amplification or contamination were not detected in water $\left(\mathrm{H}_{2} \mathrm{O}\right)$ control.

By comparison, other, similar transcription factors were expressed at lower levels and were not increased in lesions. The transcription factor CREB showed only a small increase from 3 to $4 \%$. The mRNA levels of the Sp1 transcription factor, an important competitor for Egr-1 at certain promoter domains $(4,5)$, was elevated from $2 \%$ in medial tissues to $13 \%$ in lesion tissue. Egr- 1 is the prototype member of the NGFI-A (nerve growth factor inducible-type A) family of transcription factors. The NGFI-B family of factors may also have transcription factor activity, but includes nuclear orphan receptors similar to thyroid and steroid receptors. This NGFI-B family includes the TR3 factor, which was contained within the same arrays and was expressed at low levels that did not differ between lesion and media (Figure 1b).

Confirmation of Egr-1 $m R N A$ expression by RT-PCR analysis. Because the cDNA arrays depend upon hybridization of labeled cDNA reverse transcripts to immobilized capture probes (averaging 300 nucleotides), the potential for nonspecific hybridization exists. Whereas the high stringency of the hybridization and washing should suppress nonspecific hybridization, the mRNA from 4 addition-

\section{Figure 3}

Analysis of mRNA for Egr-1 in IMA versus atherosclerotic carotid arteries. IMA were acquired from patients undergoing CABG. The mRNA was prepared from tunica media of 4 patients (31-34) and, for reference, compared with reamplification of E217 media and lesion, as shown in Figure 2. Primers were directed at Egr-1 or $\beta$-actin for normalization. Nonspecific amplification or contamination were not detected in water $\left(\mathrm{H}_{2} \mathrm{O}\right)$ control. al patients, not included in the array analysis, was prepared under highly controlled conditions and analyzed by RT-PCR. Consistently higher levels of amplimers for Egr-1 (550 bp) were observed in the lesion (L) than in the adjacent media $(\mathrm{M})$ of the same patient (Figure 2a, 2 patients shown). The amount of mRNA used for amplification was identical in both tissues, a fact confirmed by a separate RT-PCR for RhoA. The difference in Egr-1 mRNA levels was semiquantitated in these samples by serial dilution of the lesion $(\mathrm{L})$ sample until it reached a level similar to the media (M). As shown in Figure $2 b$, the E213 lesion sample could be diluted 4-fold before it was comparable to the media levels, and the E217 sample could be diluted 8-fold before comparable levels were achieved, thus supporting the 5 -fold difference observed in the arrays. The Egr-1 amplimer was sequenced with 4color dye-terminator methods and found to match identically with the Egr-1 cDNA sequence (M62829) over 300 bp of the accurately readable region. These studies demonstrate that Egr-1 transcript expression was detected at a 5-fold higher level in lesions than in the adjacent media and confirm the findings from the genomic expression arrays.

Effect of ex vivo duration on Egr-1 expression. Because Egr1 is an early response gene, the induction of Egr- 1 expression could have occurred ex vivo, as a result of stress resulting from vascular surgery and postoperative dissection. To determine whether the interval between surgical excision of the specimen and the preparation of RNA affected Egr-1 induction, patient specimens were prepared under conditions that allowed the rapid dissection and fixation of the tissue (5 minutes) whereas identical portions of tissue were allowed to incubate under conditions similar to the array experiments (maximally 65 minutes). The results are shown in Figure 2a, which indicates that regardless of whether the RNA was derived from lesion (L) or media (M), Egr-1 levels did not increase in the ex vivo conditions used to transport the specimens from the surgical suite to the laboratory. Rather, Egr-1 levels tended to decrease slightly, though the differences between media and lesion remain. Furthermore, if the Egr-1 levels were induced ex vivo, then both the lesion and media tissues should be induced similarly, which is not observed.

Expression of Egr-1 mRNA in lesions versus internal mammary arteries. The medial portions of the endarterectomy specimens are a useful control for the adjacent atherosclerotic lesion, but typically are not histologically

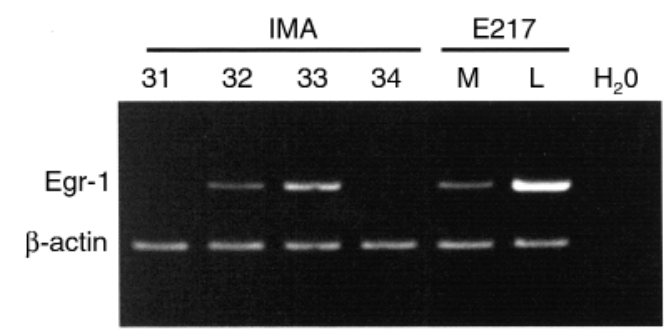




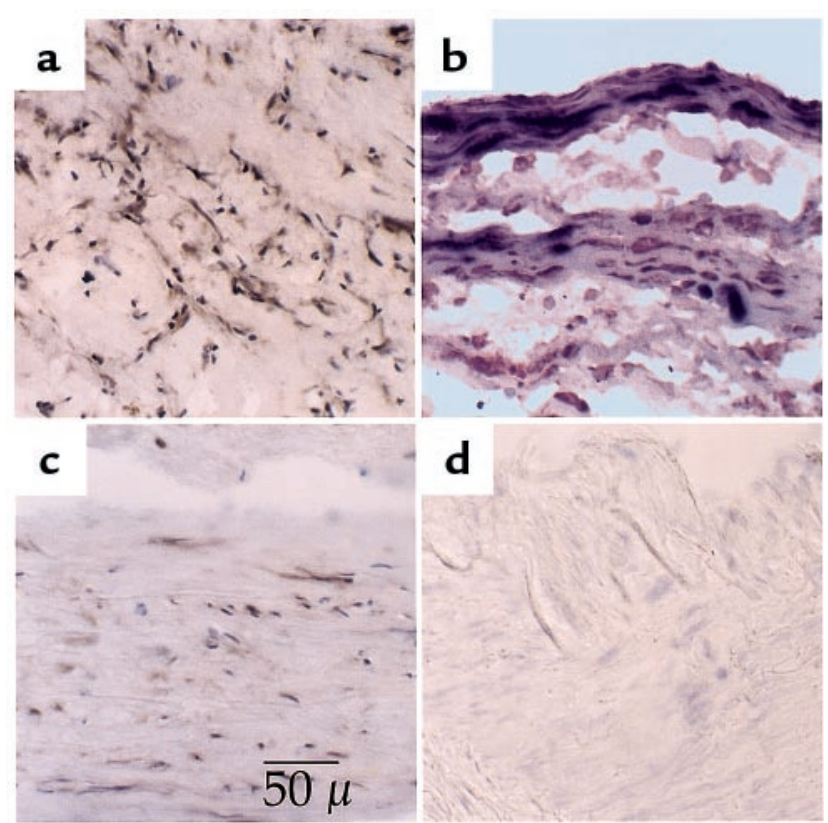

Figure 4

Immunochemical identification of Egr-1 in human atherosclerotic carotid arteries and IMA. Frozen sections of human endarterectomies or IMA were probed with Egr-1-specific antibodies. (a) Atherosclerotic carotid artery lesion stained with anti-Egr-1 antibodies yielding a dark brown reaction product in positive cells. (b) Doublestaining reveals strong Egr-1 staining (brown) in lesion cells of the fibrous cap that co-stain with the SMC/myofibroblast marker $\alpha$ actin (dark blue). Egr-1 staining is also visible in cells that stain only faintly for $\alpha$-actin. (c) Adjacent tunica media from the same section showing sparse labeling. (d) Egr-1 staining in frozen sections from IMA obtained during bypass grafting. Only a light hematoxylin stain (blue) is visible, whereas Egr-1 staining (brown) is not detected.

normal vessels by virtue of their proximity to an occlusive, fibroproliferative lesion. To determine Egr-1 expression in a less-diseased vessel, normal internal mammary arteries (IMA) were acquired from CABG, and RNA was prepared as rapidly as possible given different surgical conditions (average 15 minutes). RNAs from 4 patients were analyzed by RT-PCR for Egr- 1 and compared with RNA from E217 media and lesion (Figure 2), which was reamplified under identical conditions. As shown in Figure 3, E217 lesion RNA again showed higher levels of Egr-1 expression than the adjacent media, and levels in the IMA ranged from undetectable to just higher than the E217 media. The presence of amplifiable mRNA was confirmed by parallel PCR reactions for $\beta$-actin. Thus, there appears to be less Egr-1 transcript in normal IMA than in vascular lesions.

Expression of Egr-1 antigen in human tissues by immunocytochemistry. To determine the pattern of Egr-1 expression in the atherosclerotic lesion, as well as the cell types involved, both carotid lesions and IMA were immunostained with an Egr-1-specific antibody. A total of 12 carotid lesions were examined as frozen sections (Figure 4). Staining in the lesion was strong in 9 of 12 patients with principal involvement of the $\alpha$-actin-expressing SMC-like cells (Figure 4a). In contrast, the adjacent media, when present, showed much lower expression of Egr-1, though positive cells could be identified (Figure 4c). Regions of endothelial cells covering the lesion were occasionally positive for Egr-1. Double staining of macrophages with HAM56 and Egr-1 indicated that macrophages could be positive for Egr-1, but that Egr-1 staining was typically intense in the SMC-like cells surrounding the macrophages (not shown). Double-staining with $\alpha$-actin, a SMC/myofibroblast marker, and Egr-1 indicated that the majority of Egr-1-positive cells were also $\alpha$-actin positive (Figure 4b).

In contrast to the carotid lesions, the IMA showed much lower immunoreactivity. Five of 6 arteries showed no detectable staining for Egr-1 (Figure 4d), whereas 1 artery showed weak and diffuse staining in the medial SMC. Consistent with the RT-PCR findings, Egr-1 staining in the IMA was lower, or at best, similar to the weak, sparse staining of the carotid media shown in Figure 4c.

Expression of $m R N A$ s for Egr-1-inducible genes in atherosclerotic lesions. To determine whether the Egr-1 was transcriptionally active within the lesion cells, the expression of Egr-1 responsive genes was evaluated. Of the known or suspected Egr-1-responsive genes, 13 were contained within the cDNA arrays (Figure 5). In 12 of the 13 genes, the levels of Egr-1 RNA were higher in the lesion than in the adjacent media. In some cases, the difference was dramatic: CD44 was essentially undetectable within the media, but was expressed at $15 \%$ of the level of housekeeping genes in the lesion. ICAM- 1 was expressed at $3 \%$ in media and $78 \%$ in the lesion, a 26 -fold induction.

Several cytokines and growth factors are also mem-

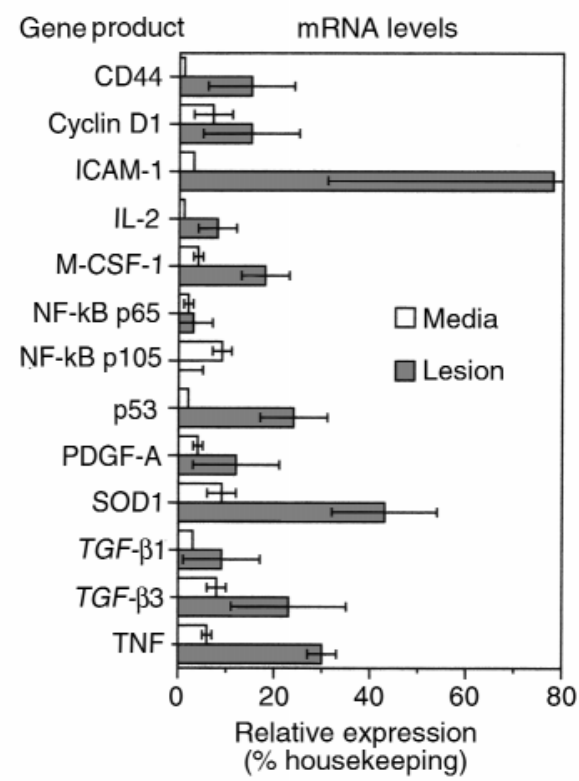

\section{Figure 5}

The mRNA levels of Egr-1-responsive genes in the human lesion versus adjacent media. Human carotid artery lesions were dissected and mRNA was prepared as in Figure 1. The cDNA arrays were examined for the expression of Egr-1-inducible genes with the expression level expressed as a percent of housekeeping genes, as shown in Figure 1. Error bars, SEM; $n=3$ experiments. 


\section{Figure 6}

Egr-1 expression in aortae of LDLR-deficient mice fed a high-cholesterol diet. $\mathrm{LDLR}^{-1-}$ mice were fed a diet containing $1.25 \%$ cholesterol for up to 20 weeks before sacrifice. At sacrifice, the mice were perfusion fixed, and the aortae were sectioned in OCT. Sections were immunostained for Egr1 , and counterstained with hematoxylin, as in Figure 4. (a) Photomicrograph of an aortic section before cholesterol feeding. (b-d) Photomicrographs of aortic sections harvested after 5,10 , and 20 weeks, respectively, of cholesterol feeding. (e) Aorta stained with Egr-1 antibody preblocked with an excess of peptide antigen. (f) Aorta stained with irrelevant purified IgG.
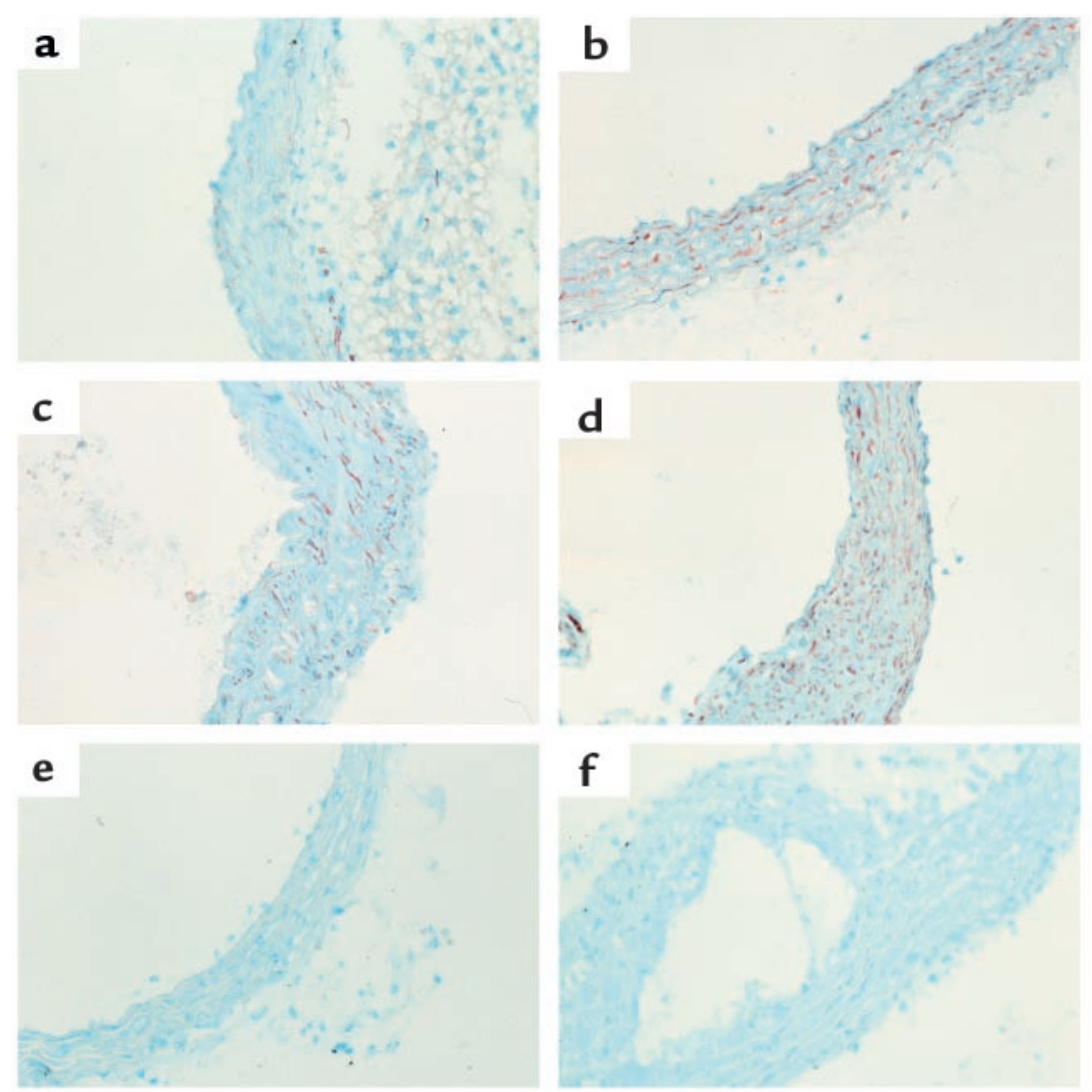

bers of the Egr-1 target gene family. Elevated levels of TNF- $\alpha$ (4-fold), IL-2 (16-fold), and M-CSF-1 (5-fold) transcripts were observed within the lesion relative to the media of the atherosclerotic vessel (Figure 5). PDGF-A chain was elevated 4-fold in the lesion from 3 to $12 \%$. Both the $\alpha$ and $\beta$ receptors for PDGF were also elevated by approximately 5 -fold each (not shown), though their promoters are not known to be controlled by Egr-1. TGF- $\beta 1$ and TGF- $\beta 3$, both containing multiple predicted Egr-1 sites in their promoters $(8,21)$, were elevated in the lesion.

Egr-1-dependent genes may also include those whose products influence cell survival or provide protective functions. Levels of human copper-zinc superoxide dismutase ( $\mathrm{Cu}, \mathrm{Zn}$-SOD) were increased in the lesions relative to the media about 5-fold (Figure 5). Analysis of the genomic arrays reveals that levels of p53 transcripts are increased in the lesion, relative to the media (Figure 5). In contrast, transcript levels of other transcription factors capable of regulating cell survival, such as the $\mathrm{p} 65$ subunit of NF- $\mathrm{KB}$, were expressed at a low level in the media (2\%), with a small increase in the lesion (3\%). The p105 precursor to p50, a putative Egr-1-responsive gene (22), was expressed in media (9\%) but not the lesion $(0 \%)$, and is thus inconsistent with complete control by Egr-1. The fact that some putative Egr-1-inducible genes are not increased in the lesion is reassuring and suggests that differences are gene specific and not the result of global changes or systematic errors in the array methodology. Thus, Egr-1 is induced in atherosclerotic lesions and some genes with previously characterized Egr-1 elements are also upregulated, though cause and effect cannot be established from this type of analysis.

Analysis of Egr-1 expression during hypercholesterolemiainduced atherosclerosis in the $L D L R^{-/-}$mouse. The preceding sections demonstrate that Egr-1 transcript and protein can be identified in end-stage, human carotid artery lesions. To examine the relationship of Egr-1 to the early development of lesions, the expression of the transcription factor in the vessel wall of homozygous LDLR $^{-/-}$mice was examined immunocytochemically. The $\mathrm{LDLR}^{-/}$mice fed a normal chow diet have only a modest elevation in plasma cholesterol and do not develop lesions (20). However, when fed a cholesterolrich diet, these mice develop complex lesions of atherosclerosis similar to those in humans (23).

To determine the pattern of Egr-1 expression in the $\mathrm{LDLR}^{-/-}$model of atherosclerosis, mice were placed on the cholesterol-rich diet for $0,5,10$, or 20 weeks. These time points were selected based on preliminary studies that demonstrated lesions begin to develop in the aortic arch and near the ostia after about 4 weeks on the diet. Sections of the aorta were immunostained with an Egr-1-specific antibody, as shown in Figure 6. In control animals there is little detectable expression of Egr1 antigen (Figure 6a). Egr-1 can be detected in vascular SMC in the aorta after 5 weeks (Figure 6b), and there is a progressive increase in Egr-1 expression in the SMC 
at 10 and 20 weeks on the diet (Figure 6, c and d, respectively). In control studies, staining with the anti-Egr-1 antibody could be blocked by an excess of the Egr-1 peptide to which the antibody was raised (Figure 6e), and irrelevant purified IgG did not stain the sections (Figure 6f).

Expression of Egr-1 in atherosclerotic lesions formed at 20 weeks is shown in Figure 7. Egr-1 antigen can be detected in vascular SMC adjacent to the developing plaque (Figure 7a), as well as within cells found near the necrotic core (Figure 7b). However, little expression was detected in the macrophage-rich core. Thus, hypercholesterolemia-associated upregulation of Egr1 expression suggests a role for this transcription factor in lesion formation.

\section{Discussion}

The lesions of atherosclerosis are thought to result from an excessive fibroproliferative response to multiple forms of insult to the vessel wall (19). To identify similarities and differences between the SMC-like cells of the lesion versus the "true" SMC of the arterial intima and media, these tissues were analyzed by cDNA expression arrays. Transcript profiling revealed that several genes were differentially regulated in the diseased vessels, most notably the transcription factor Egr-1. Multiple approaches were used to confirm that the induction of Egr-1 was valid: first, RT-PCR analysis of the same and similar tissues confirmed the findings from the arrays; second, elevated Egr-1 protein was detected by immunostaining in the atherosclerotic lesions; third, Egr-1 induction was observed in a mouse model of atherosclerosis; and finally, the expression of Egr-1-inducible genes was elevated in the same arrays, suggesting that Egr1 induction led to transcription of Egr-1-responsive genes. All of these approaches suggest that the induction of Egr-1 and Egr-1-responsive genes in the human lesion is strong, reproducible, and potentially significant to the etiology of atherosclerosis.

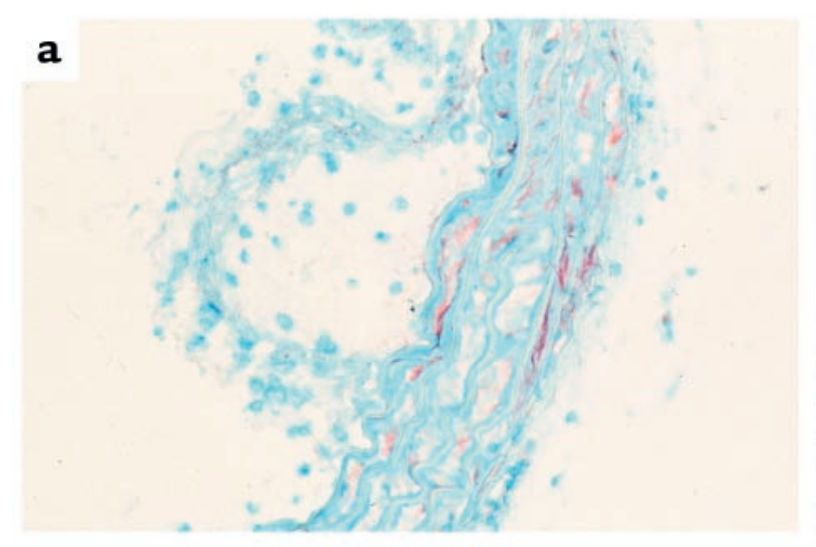

There are certain inherent limitations to the expression analysis of RNAs from complex tissues like the atherosclerotic plaque. Foremost is that the lesion may contain multiple cell types that would differ from patient to patient. By focusing on the fibrous cap of the lesion, the present analysis would minimize, though not eliminate, the contribution of $\mathrm{T}$ cells and macrophages in the lesion because the majority of these cells tend to localize to "shoulder" regions and the fibro-fatty core of the plaque, both of which were intentionally trimmed at the same time that the endothelium was scraped.

The high level of Egr-1 in lesions could be viewed as surprising because the atherosclerotic plaque has a relatively low mitotic index, and the normal media has an even lower mitotic rate. In human carotid artery tissue acquired by endarterectomy, BrdU incorporation indicated that between 3 and $15 \%$ of the cells in the lesion might be proliferating, and many of these cells were identified as subendothelial macrophages (24). Considering that the immunocytochemistry indicated that the majority of the SMC-like cells were expressing elevated Egr-1, it would seem likely that the Egr-1 is expressed by nonproliferating cells that are in an activated state.

Egr-1 is readily induced by shear stress and laminar flow disturbances (25), mechanical injury, hypoxia, reactive oxygen species, and growth factors such as FGF and PDGF (3). Of particular relevance to atherosclerosis is the recent observation that both native and oxidized lipoproteins induce tissue factor gene expression in SMC through Egr-1 and Sp1 (26). Each of these stimuli might be relevant initiators of Egr-1 expression within the atherosclerotic lesion. Reactive oxygen species, in conjunction with released growth factors, might explain the locally high levels of Egr-1 expression in the SMC surrounding macrophage-rich regions of the plaque.

The induction of Egr-1 after endothelial injury in vitro appears to be due to local release of FGF-2 (17). In human atheroma, FGF-2 is readily expressed in the lesion (27), and infusions of FGF-2 markedly increase

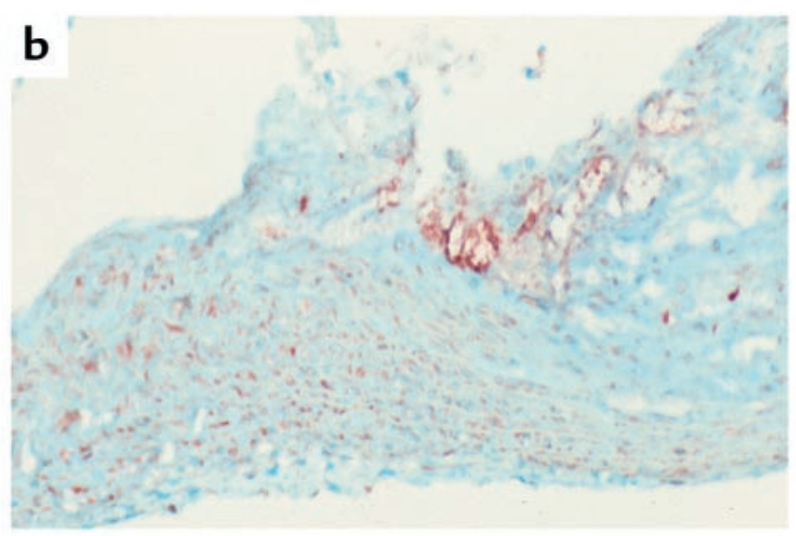

Figure 7

Egr-1 expression in the late lesions of LDLR ${ }^{-/-}$mice fed a cholesterol-rich diet. LDLR ${ }^{-/}$mice sacrificed at 20 weeks, were stained with Egr-1 antibodies. Regions containing raised atherosclerotic lesions are shown in (a) and (b) with Egr-1-positive cells producing a red reaction product (Fast Red) against the light-blue/green counterstain (methylene green). 
intimal thickening after balloon injury of the rat (28). Likewise, NGF and its receptors are expressed in the human atherosclerotic lesion and are increased in response to vascular injury (29). Like PDGF, neurotropins have chemotactic actions on SMC and, in light of the current data, may augment expression of Egr-1 in the vascular lesion. Transcriptional control of Egr-1 expression appears to be positively regulated by a single, proximal serum response element (SRE) and a NF-KB-like negative regulatory element (30).

Egr-1 activity is probably closely regulated under normal conditions. Egr-1 can associate with corepressor proteins that can diminish the transcription of multiple target genes. These proteins include Egr-1/NGFI-A binding corepressors NAB1 and NAB2 (31-33). NAB2 is rapidly and transiently expressed in vascular SMC in response to mechanical injury or growth factors such as FGF-2 (34). These corepressors may be part of a negative feedback mechanism that serves to downregulate Egr-1-mediated gene transcription in injured SMC.

Once Egr-1 is induced, it appears capable of both positive and negative transcriptional regulation of a variety of critical stress/injury response genes. In the present analysis, 13 genes with known or suspected Egr-1 responses were included within the cDNA expression arrays. All but 1 of these genes (NF- $\mathrm{KB}, \mathrm{p} 105 / \mathrm{p} 50$ subunit), followed an expression pattern that paralleled the expression of Egr-1. Whereas the present analysis is purely correlational, it demonstrates this correlation between Egr-1 and its target genes in human vascular disease. Stronger evidence of the relationship between Egr-1 and target gene induction has already been provided for many of the genes by using reporter genes and supershift binding assays.

Growth factors are one of several targets of Egr-1. In vitro studies have shown that Egr-1 binds to Egr-1 recognition sites within the promoter of both the PDGF-A (6) and the PDGF-B chain (4), resulting in induction of the mRNAs. In the present studies, PDGF-A was elevated almost 4-fold, consistent with previous reports demonstrating elevated PDGF-A in the lesion myofibroblasts (35). PDGF-B chain was not included in the arrays, though previous publications have indicated increased PDGF-B mRNA in the myofibroblasts of human carotid lesions compared to the adjacent media (35).

Another putative Egr-1-inducible growth factor gene is TGF- $\beta 1$. Both TGF- $\beta 1$ and TGF- $\beta 3$ contain predicted Egr-1-binding sites in their promoters, and both mRNAs were elevated 3-fold in the fibrous cap of the lesion relative to the adjacent media. Previous studies have documented that both human (36) and animal (37) vascular lesions overexpress TGF- $\beta 1 \mathrm{mRNA}$ and protein and that inhibition of TGF- $\beta 1$ reduces the vascular fibroproliferative response to injury in rats (38). Active TGF- $\beta 1$ and its 2 receptors are expressed focally in these late atherosclerotic lesions and particularly in the fibrous cap of the lesion (39). The Egr-1 induction of PDGFs and TGF- $\beta$ s would contribute to SMC/myofibroblast migration, proliferation, and extracellular matrix accumulation that are the hallmarks of advanced atherosclerosis.

The induction of other cytokines by Egr-1 would control the influx of inflammatory cells and their subsequent activation. Previous studies have defined functional Egr1 sites in the promoters of the TNF- $\alpha$ (9) and IL-2 genes (40-42). M-CSF-1 is predicted to be an Egr-1 target gene on the basis of promoter sequence (43), but direct experimental verification is absent presently. The increase in TNF- $\alpha$ in the lesion may activate the endothelial and SMC and play a role in regulating the survival of cells in the lesion (44). The increased production of IL-2 and M-CSF1 may prolong the survival of recruited lymphocytes and macrophages, respectively. ICAM- 1 has been shown previously to be expressed at high levels in endothelial cells, macrophages, and SMCs of the human atherosclerotic lesions (45), as well as in rabbit and mouse vascular lesions (46). Egr-1-dependent induction of ICAM-1, in conjunction with cytokines and growth factors, may also play a role in the recruitment of cells to the lesions.

Other Egr-1-dependent genes include those whose products may further influence cell survival in the atherosclerotic lesion. One of these target genes is the tumor suppressor protein, $\mathrm{p} 53$, an essential regulatory molecule in cell proliferation and apoptosis. This tumor suppressor is an essential molecule in cell proliferation and apoptosis and has been postulated to play a part in the development of atherosclerosis. In the present studies, p53 mRNA was elevated almost 12-fold in the lesion, a finding supported by previous studies that observed elevated p53 immunoreactivity in human atherosclerotic lesions compared with IMA (47).

In the apoE-null mouse model of atherogenesis, the absence of p 53 accelerates atherosclerosis by increasing cell proliferation in vivo, although p53-independent mechanisms appear to mediate apoptosis (48). Interestingly, in a human tumor cell line, Egr-1 counteracted apoptosis by the coordinated activation of TGF $\beta-1$, fibronectin, the cyclin-dependent kinase inhibitor, p21, and focal adhesion kinase, leading to enhanced cell attachment and reduced caspase activity (49). The survival effect of Egr-1 in this tumor line was dominant over the apoptotic effect of p53. Thus, Egr-1 may coordinate the expression gene products important for survival of vascular cells in atherosclerotic lesions.

Egr-1-dependent gene expression may also play a role in the control of oxidant stress in atherosclerotic lesions. SOD1 participates in the control of intracellular concentrations of reactive oxygen intermediates, and a functional Egr-1 element has been defined in the proximal promoter of the gene (50). Thus, Egr-1 responsiveness to reactive oxygen species leads to induction of SOD1, thereby completing a corrective feedback loop to control free-radical damage to the cell.

Egr-1 is commonly found to bind to promoter regions overlapping consensus $\mathrm{Sp} 1$ sites that can either facilitate or suppress transcription (51). In addition to the genes discussed above, known Egr-1-inducible genes include apoAI (52), 5-lipoxygenase (53), thymidine kinase (54), and the multidrug-resistance gene 
MDR1 (55), although these genes were not contained within the arrays. Other genes, however, are thought to be suppressed by Egr-1, presumably by displacement of $\mathrm{Sp} 1$. For example, insulin-induced suppression of malic enzyme gene transcription appears to be mediated by Egr-1 (56). Likewise, B-cell clones overexpressing Egr-1 show decreased expression of fas (CD95) and $\mathrm{CD} 23$, which makes them functionally resistant to fasmediated apoptosis (57).

Given that Egr- 1 is a stress-responsive gene, the present data indicates that the SMC/myofibroblast cells of the human and mouse lesions exist in a highly activated state, as opposed to passively accumulating in the intima. The elevations of Egr-1 in the human carotid lesion indicate that previous in vitro and in vivo studies implicating Egr- 1 in the vascular response to injury can reasonably be extended to the human disease. Elevated Egr-1 expression in the lesion was associated with elevations in a wide spectrum of Egr-1-responsive genes that would be relevant to atherogenesis. As suggested by the recent in vivo studies of Santiago et al. (18), it is not unreasonable to speculate that interference with Egr-1 induction or function might prevent the negative sequelae of vascular injury after revascularization procedures such as angioplasty and endarterectomy.

\section{Acknowledgments}

This work is dedicated to the memory of Russell Ross, who, despite his passing, remains a vital part of atherosclerosis research. He was an insightful scientist, a warm friend, and mentor to many of us. The authors are grateful for the financial support of the National Institutes of Health through the National Heart, Lung and Blood Institute's (NHLBI) Specialized Centers of Research (SCORs HL56985 and HL56987) in Molecular Mechanisms of Atherosclerosis and through the National Institutes on Aging (NIA) to T. McCaffrey (AG12712) and NHLBI to T. Collins (R37-HL35716).

1. Gashler, A., and Sukhatme, V.P. 1995. Early growth response protein 1 (Egr-1): prototype of a zinc-finger family of transcription factors. Prog. Nucleic Acid Res. Mol. Biol. 50:191-224.

2. Khachigian, L., and Collins, T. 1997. Inducible expression of Egr-1dependent genes: a paradigm of transcriptional activation in vascular endothelium. Circ. Res. 81:457-461.

3. Silverman, E., and Collins, T. 1999. Pathways of Egr-1-mediated gene transcription in vascular biology. Am. J. Pathol. 154:665-670.

4. Khachigian, L., Williams, A., and Collins, T. 1995. Interplay of Sp1 and Egr-1 in the proximal platelet-derived growth factor A-chain promoter in cultured vascular endothelial cells. J. Biol. Chem. 270:27679-27686.

5. Silverman, E.S., Khachigian, L.M., Lindner, V., Williams, A.J., and Collins, T. 1997. Inducible PDGF A-chain transcription in smooth muscle cells is mediated by Egr-1 displacement of Sp1 and Sp3. Am.J. Physiol. 273:H1415-H1426.

6. Khachigian, L., Lindner, L., Williams, A., and Collins, T. 1996. Egr1induced endothelial gene expression: a common theme in vascular injury. Science. 271:1427-1431.

7. Biesiada, E., Razandi, M., and Levin, E.R. 1996. Egr-1 activates basic fibroblast growth factor transcription. Mechanistic implications for astrocyte proliferation. J. Biol. Chem. 271:18576-18581.

8. Kim, S.-J., Jeang, K.-T., Glick, A.B., Sporn, M.B., and Roberts, A.B. 1989. Promoter sequences of the human transforming growth factor- $\beta 1$ gene responsive to transforming growth factor- $\beta 1$ autoinduction. J. Biol. Chem. 264:7041-7045.

9. Kramer, B., Meichle, A., Hensel, G., Charnay, P., and Kronke, M. 1994. Characterization of an Krox-24/Egr-1-responsive element in the human tumor necrosis factor promoter. Biochim. Biophys. Acta. 1219:413-421.

10. Maltzman, J.S., Carmen, J.A., and Monroe, J.G. 1996. Transcriptional regulation of the ICAM-1 gene in antigen receptor- and phorbol esterstimulated B lymphocytes: role for transcription factor EGR1. J. Exp. Med. 183:1747-1759.

11. Maltzman, J.S., Carman, J.A., and Monroe, J.G. 1996. Role of EGR1 in regulation of stimulus-dependent CD44 transcription in B lymphocytes. Mol. Cell. Biol. 16:2283-2294.

12. Nair, P., et al. 1997. Early growth response-1-dependent apoptosis is mediated by p53. J. Biol. Chem. 272:20131-20138.

13. Cui, M.Z., et al. 1996. Transcriptional regulation of the tissue factor gene in human endothelial cells is mediated by Sp1 and EGR-1. J. Biol. Chem. 271:2731-2739.

14. Verde, P., Boast, S., Franze, A., Robbiati, F., and Blasi, F. 1988. An upstream enhancer and a negative element in the $5^{\prime}$ flanking region of the human urokinase plasminogen activator gene. Nucleic Acids Res. 16:10699-10716

15. Haas, T.L., Stitelman, D., Davis, S.J., Apte, S.S., and Madri, J.A. 1999. Egr-1 mediates extracellular matrix-driven transcription of membrane type 1 matrix metalloproteinase in endothelium. J. Biol. Chem. 274:22679-22685.

16. Yan, S.F., et al. 1998. Tissue factor transcription driven by Egr-1 is a critical mechanism of murine pulmonary fibrin deposition in hypoxia. Proc. Natl. Acad. Sci. USA. 95:8298-8303.

17. Santiago, F.S., Lowe, H.C., Day, F.L., Chesterman, C.N., and Khachigian, L.M. 1999. Early growth response factor- 1 induction by injury is triggered by release and paracrine activation by fibroblast growth factor-2. Am. J. Pathol. 154:937-944.

18. Santiago, F., et al. 1999. New DNA enzyme targeting Egr-1 mRNA inhibits vascular smooth muscle proliferation and regrowth after injury. Nat. Med. 5:1264-1269.

19. Ross, R. 1993. The pathogenesis of atherosclerosis: a perspective for the 1990s. Nature. 362:801-809.

20. Ishibashi, S., et al. 1993. Hypercholesterolemia in low density lipoprotein receptor knockout mice and its reversal by adenovirus-mediated gene delivery. J. Clin. Invest. 92:883-893.

21. Lafyatis, R., et al. 1990. Structural and functional characterization of the transforming growth factor beta 3 promoter. A cAMP-responsive element regulates basal and induced transcription. J. Biol. Chem. 265:19128-19136.

22. Cogswell, P.C., Mayo, M.W., and Baldwin, A.S., Jr. 1997. Involvement of Egr-1/RelA synergy in distinguishing $\mathrm{T}$ cell activation from tumor necrosis factor-alpha-induced NF-kappa B1 transcription. J. Exp. Med. 185:491-497.

23. Breslow, J.L. 1996. Mouse models of atherosclerosis. Science. 272:685-688.

24. Ito, M., et al. 1995. Expression of PDGF in relation to cell division in atherosclerotic intima of human carotid arteries. Neurol. Res. 17:345-348.

25. Nagel, T., Resnick, N., Dewey, C., and Gimbrone, M. 1999. Vascular endothelial cells respond to spatial gradients in fluid shear stress by enhanced activation of transcription factors. Arterioscler. Thromb. Vasc. Biol. 19:1825-1834.

26. Cui, M., Penn, M., and Chisolm, G. 1999. Native and oxidized low density lipoprotein induction of tissue factor gene expression in smooth muscle cells is mediated by both Egr-1 and Sp1. J. Biol. Chem. 274:32795-32802.

27. Hughes, S.E., Crossman, D., and Hall, P.A. 1993. Expression of basic and acidic fibroblast growth factors and their receptor in normal and atherosclerotic human arteries. Cardiovasc. Res. 27:1214-1219.

28. Lindner, V., Lappi, D.A., Baird, A., Majack, R.A., and Reidy, M.A. 1991 Role of basic fibroblast growth factor in vascular lesion formation. Circ. Res. 68:106-113.

29. Donovan, M.J., et al. 1995. Neurotropin and neurotropin receptors in vascular smooth muscle cells: regulation of expression in response to injury. Am. J. Pathol. 147:1-16.

30. Aicher, W., Sakamoto, K., Hack, A., and Eibel, H. 1999. Analysis of functional elements in the human Egr-1 gene promoter. Rheumatol. Int. 18:207-214.

31. Russo, M.W., Sevetson, B.R., and Milbrandt, J. 1995. Identification of NAB1, a repressor of NGFI-A- and Krox20-mediated transcription. Proc. Natl. Acad. Sci. USA. 92:6873-6877.

32. Swirnoff, A.H., et al. 1998. Nab1, a corepressor of NGFI-A (Egr-1), contains an active transcriptional repression domain. Mol. Cell. Biol. 18:512-524.

33. Svaren, J., et al. 1996. NAB2, a corepressor of NGFI-A (Egr-1) and Krox 20, is induced by proliferative and differentiative stimuli. Mol. Cell. Biol. 16:3545-3553.

34. Silverman, E., et al. 1999. Vascular smooth muscle cells express the transcriptional corepressor NAB2 in response to injury. Am. J. Pathol. 155:1311-1317. 
35. Wilcox, J.N., Smith, K.M., Williams, L.T., Schwartz, S.M., and Gordon, D. 1988. Platelet-derived growth factor mRNA detection in human atherosclerotic plaques by in situ hybridization. J. Clin. Invest. 82:1134-1143

36. Nikol, S., et al. 1992. Expression of transforming growth factor- $\beta 1$ is increased in human vascular restenosis lesions. J. Clin. Invest. 90:1582-1592

37. Majesky, M.W., Lindner, V., Twardzik, D.R., Schwartz, S.M., and Reidy, M.A. 1991. Production of transforming growth factor- $\beta 1$ during repair of arterial injury. J. Clin. Invest. 88:904-910.

38. Wolf, Y.G., Rasmussen, L.M., and Ruoslahti, E. 1994. Antibodies against transforming growth factor- $\beta 1$ suppress intimal hyperplasia in a rat model. J. Clin. Invest. 93:1172-1178.

39. McCaffrey, T., et al. 1999. The expression of TGF- $\beta$ receptors in human atherosclerosis: evidence for acquired resistance due to receptor imbalance. J. Mol. Cell. Cardiol. 31:1627-1642.

40. Skerka, C., Decker, E.L., and Zipfel, P.F. 1995. A regulatory element in the human interleukin 2 gene promoter is a binding site for the zinc finger proteins Sp1 and EGR-1. J. Biol. Chem. 270:22500-22506.

41. Lin, J.X., and Leonard, W.J. 1997. The immediate-early gene product Egr-1 regulates the human interleukin-2 receptor beta-chain promoter through noncanonical Egr and Sp1 binding sites. Mol. Cell. Biol. 17:3714-3722.

42. Decker, E.L., Skerka, C., and Zipfel, P.F. 1998. The early growth response protein (EGR-1) regulates interleukin-2 transcription by synergistic interaction with the nuclear factor of activated T cells. J. Biol. Chem. 273:26923-26930.

43. Harrington, M.A., et al. 1993. Inhibition of colony-stimulating factor1 promoter activity by the product of the Wilms' tumor locus. J. Biol. Chem. 268:21271-21275.

44. Chatterjee, S. 1998. Sphingolipids in atherosclerosis and vascular biology. Arterioscler. Thromb. Vasc. Biol. 18:1523-1533.

45. Poston, R.N., Haskard, D.O., Coucher, J.R., Gall, N.P., and JohnsonTidey, R.R. 1992. Expression of intercellular adhesion molecule-1 in atherosclerotic plaques. Am. J. Pathol. 140:665-673.
46. Iiyama, K., et al. 1999. Patterns of vascular cell adhesion molecule-1 and intercellular adhesion molecule- 1 expression in rabbit and mouse atherosclerotic lesions and at sites predisposed to lesion formation. Circ. Res. 85:199-207.

47. Ihling, C., et al. 1998. Co-expression of p53 and MDM2 in human atherosclerosis: implications for the regulation of cellularity of atherosclerotic lesions. J. Pathol. 185:303-312.

48. Guevara, N.V., Kim, H.S., Antonova, E.I., and Chan, L. 1999. The absence of $\mathrm{p} 53$ accelerates atherosclerosis by increasing cell proliferation in vivo. Nat. Med. 5:335-339.

49. de Belle, I., et al. 1999. P53 and Egr-1 additively suppress transformed growth in HT1080 cells but Egr-1 counteracts p53-dependent apoptosis. Oncogene. 18:3633-3642.

50. Minc, E., et al. 1999. The human copper-zinc superoxide dismutase gene (SOD1) proximal promoter is regulated by Sp1, Egr-1, and WT via non-canonical binding sites. J. Biol. Chem. 274:503-509.

51. Huang, R., Fan, Y., Ni, Z., Mercola, D., and Adamson, E. 1997. Reciprocal modulation between Sp1 and Egr1. J. Cell. Biochem. 66:489-499.

52. Kilbourne, E.,Widom, R., Harnish, D., Malik, S., and Karathanasis, S. 1995. Involvement of early growth response factor Egr-1 in apoliprotein AI gene transcription. J. Biol. Chem. 270:7004-7010.

53. Silverman, E., et al. 1998. Egr-1 and Sp1 interact functionally with the 5 -lipoxygenase promoter and its naturally occurring mutants. Am.J. Respir. Cell Mol. Biol. 19:316-323.

54. Molnar, G., Crozat, A., and Pardee, A. 1994. The immediate-early gene Egr-1 regulates the activity of the thymidine kinase promoter at the G0-to-G1 transition of the cell cycle. Mol. Cell. Biol. 14:5242-5248.

55. McCoy, C., Smith, D., and Cornwell, M. 1995. 12-o-tetradecanoylphorbol-13-acetate activation of the MDR1 promoter is mediated by Egr1. Mol. Cell. Biol. 15:6100-6108.

56. Barroso, I., and Santisteban, P. 1999. Insulin-induced early growth response gene (Egr-1) mediates a short term repression of rat malic enzyme gene transcription. J. Biol. Chem. 274:17997-18004.

57. Dinkel, A., et al. 1997. Transcription factor Egr-1 activity down-regulates Fas and CD23 expression in B-cells. J. Immunol. 159:2678-2684. 